\title{
Neighborhood effects in spelling in adults
}

\author{
Sébastien Roux and Patrick Bonin \\ Université Blaise Pascal, LAPSCO/CNRS UMR 6024, Clermont-Ferrand, France
}

\begin{abstract}
The participants were asked to spell aloud words for which there were either many orthographically similar words (a dense neighborhood) or few orthographically similar words (a sparse neighborhood). Words with a dense neighborhood were spelled faster and more accurately than were words with a sparse neighborhood. This finding is consistent with the hypothesis of Rapp, Epstein, and Tainturier (2002), that the cognitive spelling system has an interactive architecture incorporating feedback between individual graphemes and orthographic lexeme representations.
\end{abstract}

How do adults spell familiar words, new words, or nonwords on the basis of auditory presentation alone? It is generally assumed that spelling to dictation entails the activation of two major processing pathways or routes: a lexical and a nonlexical route (Tainturier \& Rapp, 2001). The lexical route generates the spelling of familiar auditory items by retrieving whole-word-specific spelling representations, whereas the nonlexical route builds the spelling of unfamiliar new words, or nonwords, by mapping sublexical phonological units onto sublexical orthographic units (see Figure 1). Evidence for the distinction between the two routes comes primarily from the analyses of the performance of brain-damaged patients (Rapp, 2002).

Rapp, Epstein, and Tainturier's (2002) dual-route model is one of the most highly developed models of spelling to dictation. Until now, evidence for this model has essentially been provided by cognitive neuropsychologists. Undoubtedly, this approach has been of great value for the understanding of the normal cognitive architecture involved in the spelling process. However, it is also important that this model be supported by the analysis of the performance of healthy adults and data gathered using real-time paradigms. In the present study, we investigate orthographic neighborhood effects in spelling, an issue worth investigating both theoretically and empirically. Though acknowledged as an important issue for study (Kreiner, 1996), neighborhood effects in spelling have not been investigated to the same extent as has word recognition. From a theoretical point of view, orthographic neighborhood effects are predicted by dual-route models of spelling to dictation. In particular, the finding of facilitatory effects of neighborhood would seem to provide support for the processing assumption made by Rapp et al.'s claim of feedback activation from individual graphemes to whole-word orthographic (lexeme) representations (see Figure 1). Until now, little attention has been paid to questions concerning feedback in the cognitive spelling system, and support for such a mechanism in spelling has only been provided by analyses of data ob- tained from patients. Neighborhood density refers to the number of words similar to a target word (Luce \& Pisoni, 1998). Words with many similar words are said to have a dense neighborhood, whereas words with few neighbors are said to have a sparse neighborhood. Rapp et al.'s claim predicts facilitatory effects from dense orthographic neighborhoods on both spelling speed and accuracy in normals to date; however, no such evidence is available.

\section{Rapp, Epstein, and Tainturier's (2002) Spelling Model}

Spelling a familiar word starts with the activation of its phonological lexeme in an input phonological lexicon (see Figure 1). Activation then flows to the semantic system. From there, the semantic representation activates an orthographic lexeme in an output orthographic lexicon. The orthographic lexeme allows the activation of the word's constituent graphemes. This level corresponds to an abstract letter-identity level of representation, which provides the basis for the activation of allographic representations of the intended form of written output, thus resulting in the production of writing movements. The abstract grapheme representations are used in oral spelling to activate letter-name representations.

When an unfamiliar word (or a nonword) is to be spelled, the dual-route theory assumes that a sublexical phonemeto-grapheme conversion process is involved in the production of a plausible spelling. The conversion process activates individual graphemes by exploiting sound-tospelling correspondence. More precisely, it is assumed that the sublexical conversion procedure uses high-probability phoneme-grapheme mappings (Rapp et al., 2002).

Neuropsychological evidence has been provided in support of the view that the two routes are involved in parallel in spelling. The spelling of any word is the result of output from both routes. The pattern of spelling performance of the patient L.A.T. (Rapp et al., 2002) strongly supports the hypothesis that the output of both routes is integrated

P. Bonin, patrick.bonin@univ-bpclermont.fr 


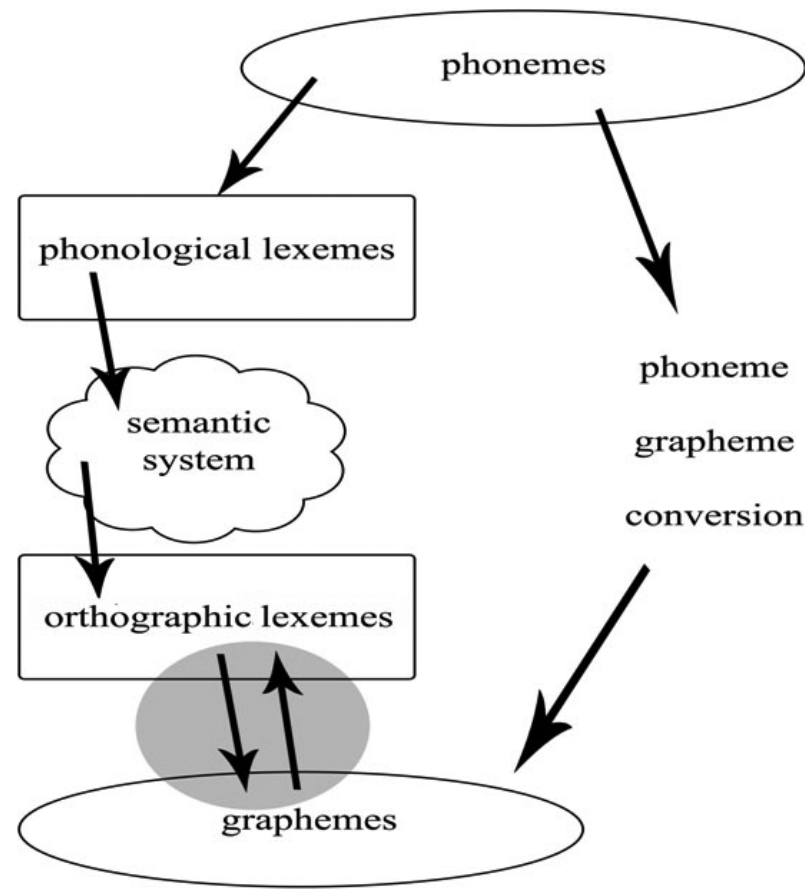

Figure 1. Architecture of the cognitive spelling system with bidirectional connections between the orthographic lexeme and grapheme levels.

at the grapheme level. When asked to spell words, L.A.T. made many phonologically plausible errors (PPEs) that contained both high- and low-probability mappings (e.g., knowledge spelled knolige). Since the nonlexical route makes use of high-probability mappings, the presence of low-probability, lexically correct elements in L.A.T.'s PPEs supports the hypothesis that the spelling of words is the result of the integrated output of elements generated by lexical and nonlexical processes.

To account for the generation of correct outputs by the two routes, it has been assumed that in the undamaged system, lexical activation prevails (Rapp et al., 2002). This is possible, if we assume the existence of bidirectional connections between the orthographic (lexeme) and grapheme levels that serve to stabilize and amplify lexical contributions over nonlexical ones (Figure 1). For instance, when spelling the word knowledge, the activation of the grapheme $e$ for the phoneme $i$ is reinforced by the feedback loop between the lexeme of the word knowledge and the graphemes that comprise it.

The error pattern of a brain-damaged patient (C.M.) has been taken as evidence that the cognitive system for spelling to dictation includes feedback connections from grapheme representations to orthographic lexeme representations (McCloskey, Macaruso, \& Rapp, 2006). The patient's spelling responses showed a letter persistence effect; that is, letters from prior spelling responses intruded into subsequent responses at rates far greater than expected by chance. Also, there was a large proportion of form lexical errors (e.g., "arm" $\rightarrow$ "amber") that originated in lexical selection rather than being "chance" lexical errors, and these errors exhibited the letter persistence ef- fect. Thus, in the production of amber instead of arm, the grapheme representations $b$ and $e$ activated for preceding targets (e.g., bench) sometimes persisted abnormally in an activated state instead of being deactivated after the response was completed.

To account for the letter persistence effect in lexical errors, it is assumed that persisting activation of letter representations at the grapheme level influences the activation of orthographic lexemes, since the connections between the orthographic lexeme and grapheme levels are bidirectional (see Figure 1). In the error "arm" $\rightarrow$ "amber," the persisting activation of the graphemes $b$ and $e$ (activated during the generation of the preceding response, "bench") led to some activation of the "amber" representation at the lexeme level, and this activation contributed to the selection of the wrong representation at the lexeme level during the processing of the word arm.

The feedback mechanism helps to strengthen and favor particular orthographic representations. Cyclic feedforward and feedback processing can create positive feedback loops between an orthographic lexical representation and its individual graphemes, which further activate the lexeme via feedback, thus increasing the feedforward activation sent to the individual graphemes, and so forth. This mechanism serves to promote correct orthographic lexical responses (McCloskey et al., 2006). Likewise, by means of this mechanism, a target word with a dense orthographic neighborhood will receive activation from many similar words via the shared individual graphemic units. On the contrary, a target word with fewer neighbors will receive activation from only a small number of formally similar words via the shared graphemic units.

\section{Neighborhood Effects}

Neighborhood effects have been investigated in several lexical processing tasks, such as reading aloud or visual/ auditory lexical decision. Studies have been conducted in language perception to better characterize the kind of neighborhood underlying these effects. Likewise, the influence of different kinds of neighbors has been investigated: orthographic (e.g., Andrews, 1997; Ziegler \& Muneaux, 2007), phonological (e.g., Yates, 2005; Ziegler \& Muneaux, 2007), and phonographic (Peereman \& Content, 1997) neighbors. Also, the polarity of these effects has been the topic of much debate (Andrews, 1997). As far as spoken production is concerned, reports of neighborhood effects are relatively scarce. Neighborhood effects have been found in spoken naming (Vitevitch, 2002; Vitevitch \& Stamer, 2006). Here, also, the polarity of these effects has been found to vary as a function of language: facilitatory in spoken naming in English (Vitevitch, 2002), but inhibitory in Spanish (Vitevitch \& Stamer, 2006). As already noted, we are not aware of any real-time study conducted in adults that has investigated the issue of spelling to dictation.

In our study, the participants were required to orally spell words with either dense or sparse orthographic neighborhoods. Rapp et al.'s (2002) claim leads to the strong prediction that words from dense neighborhoods will be spelled aloud faster than will words from sparse neighborhoods. The oral spelling-to-dictation task has previously been used 
Table 1

Statistical Characteristics of the Experimental Words

\begin{tabular}{lccccc}
\hline & \multicolumn{2}{c}{ Dense } & & \multicolumn{2}{c}{ Sparse } \\
\cline { 2 - 3 } \cline { 5 - 6 } \cline { 5 - 6 } & Type & Token & & Type & Token \\
\hline Onset (C1) consistency & .98 & .99 & & .99 & .99 \\
Vowel (V) consistency & .77 & .83 & & .66 & .68 \\
Coda (C2) consistency & .88 & .84 & & .76 & .87 \\
Rime (VC) consistency & .86 & .87 & & .88 & .93 \\
Acoustic duration (in msec) & 518.13 & & 523.6 \\
Number of phonemes* & $3.95(3-5)$ & & $3.81(3-5)$ \\
Number of letters & $5(4-6)$ & & $5.26(4-7)$ \\
Number of complex graphemes & $.30(0-1)$ & & $.61(0-2)$ \\
Uniqueness point* & $4.74(4-6)$ & & $4.52(3-6)$ \\
Adult word frequency $(\log )^{*}$ & $.82(.025-1.66)$ & & $.85(.19-1.52)$ \\
Child word frequency $(\log )^{* *}$ & $1.69(.23-1.78)$ & & $1.20(.31-1.34)$ \\
Diphone frequency (log) & $2.27(2.05-3.21)$ & & $2.57(1.04-3.46)$ \\
Bigram frequency (log)* & $3.36(3.19-4.18)$ & & $3.60(2.73-4.10)$ \\
Trigram frequency $(\log )^{*}$ & $2.69(1.87-3.36)$ & & $2.67(1.16-3.76)$ \\
Orthographic $N(p<.001)$ & $9.56(8-15)$ & & $5.52(1-6)$ \\
Phonographic $N(p<.001)$ & $5.61(2-5)$ & & $0.65(0-1)$ \\
Phonological $N$ & $13.34(2-31)$ & & $11.00(5-26)$ \\
Onset frequency*** & $1.97(1.59-2.14)$ & & $1.87(1.17-2.14)$ \\
\hline Note-Consinn & & & & &
\end{tabular}

Note-Consistency scores from LEXOP (Peereman \& Content, 1999) by type (and by token); orthographic $N$, number of orthographic neighbors as defined by Coltheart, Davelaar, Jonasson, and Besner (1977) taken from LEXOP (Peereman \& Content, 1999); phonographic $N$, number of phonographic neighbors; phonological $N$, number of phonological neighbors, from LEXIQUE (New, Pallier, Ferrand, \& Matos, 2001); onset frequency taken from VoCoLex (Dufour, Peereman, Pallier, \& Radeau, 2002). Where not shown, $p$ values are nonsignificant. ${ }^{*}$ Taken from LEXIQUE (New et al., 2001). ${ }^{* *}$ Taken from MANULEX (Lété, Sprenger-Charolles, \& Colé, 2004). $\quad$ ***From VoCoLex (Dufour et al., 2002).

in normals (e.g., Kreiner, 1996). This task is assumed to involve the activation of abstract orthographic representations and individual graphemes taken from the graphemic buffer and converted into letter-name representations (Rapp, 2002). A shadowing task was used as a control experiment. In effect, since both the oral spelling and shadowing tasks share the perceptual identification and comprehension stages, any difference between sparse and dense items due to uncontrolled difficulties arising at these stages should be reflected in both spelling and shadowing latencies.

\section{METHOD}

\section{Participants}

Sixty undergraduate psychology students at Blaise Pascal University took part in this experiment ( 30 in the spelling task and 30 in the control shadowing task). They were all native French speakers with no known hearing problems.

\section{Stimuli}

Forty-six French monosyllabic words were selected from the LEXOP database (Peereman \& Content, 1999). Half of them had dense orthographic neighborhoods, and the other half, sparse orthographic neighborhoods. Orthographic neighbors were operationally defined as the words of identical numbers of letters that can be generated by a single letter substitution (Coltheart, Davelaar, Jonasson, \& Besner, 1977). Among the orthographic neighbors, some were also phonological (i.e., phonographic) neighbors. Importantly, dense and sparse neighbors were matched for the number of phonological neighbors.

The experimental words were matched on several characteristics (see Table 1), including acoustic duration (in milliseconds); number of letters (and of complex graphemes, e.g., "ck" in rocket); initial letter (and letter name); initial, middle, and final phonemegrapheme consistency; log word frequency; and bigram and trigram frequency using the LEXIQUE database (New, Pallier, Ferrand, \& Matos, 2001), as well as onset frequency taken from the VoCoLex database (Dufour, Peereman, Pallier, \& Radeau, 2002). The mean statistical characteristics are listed in Table 1 . The 46 items were recorded by a male voice on a digital audio tape (Sony) with SoundEdit 16 (version 2).

\section{Apparatus}

The stimuli were presented through headphones (Sony MDR-A 106 LP). A microphone (ATR 20) was used as a voice key. The stimulus presentation and randomization were controlled with a Macintosh (iMac 0S9) running the PsyScope 1.2.5 software (Cohen, MacWhinney, Flatt, \& Provost, 1993).

\section{Procedure}

Participants were tested in a quiet room. Each trial began with a fixation point $\left(^{*}\right)$ printed in the middle of the screen for $500 \mathrm{msec}$, followed by the stimulus presentation through headphones. The participant had to spell the stimulus aloud as quickly as possible by speaking into the microphone. Oral spelling latencies were measured to the nearest millisecond from stimulus onset. The intertrial interval was set to $4,000 \mathrm{msec}$. The stimuli were presented in different random orders for each participant. The experiment started with nine warm-up trials. In the control shadowing task, the procedure was the same, except that the participant had to repeat each word instead of spelling each aloud.

\section{RESULTS}

Latencies corresponding to spelling errors (including phonologically plausible errors and orthographic errors) and to unexpected noises or technical errors were set apart. In addition, latencies longer than $2,000 \mathrm{msec}$ or shorter than $200 \mathrm{msec}$ were excluded. Thus, $8 \%$ of the data were discarded.

The ANOVAs were performed on oral spelling latencies and error rates. Because response latencies were measured from the onset of auditory presentations, ${ }^{1}$ they are expected to vary as a function of stimulus duration. To take variability in auditory word duration into account, item analyses were performed using stimulus duration as a covariate. Since the pattern of results was the same, we report only the analyses from the ANOVAs without the covariate.

Table 2

Mean Latencies, Their Standard Deviations (SDs, in Milliseconds), and Percentages of Errors As a Function of Orthographic Neighborhood Density and Task

\begin{tabular}{|c|c|c|c|c|c|c|c|c|c|c|c|}
\hline \multicolumn{6}{|c|}{ Oral Spelling } & \multicolumn{6}{|c|}{ Shadowing } \\
\hline \multicolumn{3}{|c|}{ Dense } & \multicolumn{3}{|c|}{ Sparse } & \multicolumn{3}{|c|}{ Dense } & \multicolumn{3}{|c|}{ Sparse } \\
\hline$M$ & $S D$ & $\%$ & $M$ & $S D$ & $\%$ & $M$ & $S D$ & $\%$ & $M$ & $S D$ & $\%$ \\
\hline 1,133 & 63 & 2 & 1,203 & 90 & 7 & 891 & 138 & 2 & 885 & 131 & 2 \\
\hline
\end{tabular}


Oral spelling latencies were shorter for words with a dense orthographic neighborhood than for words with a sparse neighborhood (see Table 2). The facilitatory neighborhood density effect $(+60 \mathrm{msec})$ was significant for both participants and items $\left[F_{1}(1,29)=24.93, M S_{\mathrm{e}}=49,679\right.$, $\left.p<.001 ; F_{2}(1,44)=5.31, M S_{\mathrm{e}}=31,789, p<.05\right]$.

Three different kinds of analyses were conducted on the errors: (1) formal errors (i.e., word substitution errors) and phonologically plausible (PP) spelling errors, (2) formal errors only, and (3) technical errors only. On formal and PP errors, the effect of neighborhood was significant $\left[F_{1}(1,29)=9.79, M S_{\mathrm{e}}=16,017, p<.01 ; F_{2}(1,44)=7.51\right.$, $\left.M S_{\mathrm{e}}=20,891, p<.01\right]$; see Table 2. It was also the case when only formal errors $\left[F_{1}(1,29)=8.70, M S_{\mathrm{e}}=1.724\right.$, $\left.p<.01 ; F_{2}(1,44)=7.04, M S_{\mathrm{e}}=2.777, p<.05\right]$ or PP errors were considered but only on participants $\left[F_{1}(1,29)=\right.$ $7.60, M S_{\mathrm{e}}=.00335, p<.01 ; F_{2}(1,44)=1.50, M S_{\mathrm{e}}=$ 0.0013 , n.s.]. Indeed, the percentages of PP errors were very small in comparison with formal errors (PP, dense $=$ $0.25 \%$ of the data, sparse $=1.6 \%$; formal, dense $=2.17 \%$, sparse $=6.52 \%$ ). Finally, when only technical errors were considered, the effect of neighborhood was not significant $(F \mathrm{~s}<1)$.

In the control shadowing task, the effect of neighborhood was significant neither on latencies nor on error (all $F$ s $<1$ ); see Table 2 .

\section{DISCUSSION}

The participants had to spell aloud words with a dense or a sparse orthographic neighborhood. The outcome was straightforward. A facilitatory effect of neighborhood was found, with words having a dense neighborhood being produced faster and more accurately than words having a sparse neighborhood. To the best of our knowledge, the present study is the first to report such a finding in an experiment using a real-time paradigm in normals and experimental stimuli selected under stringent criteria. The theoretical implication of the present finding is also straightforward, since it provides an additional constraint in the building of models of spelling. In particular, neighborhood effects are consistent with the hypothesis of bidirectional connectivity between orthographic lexemes and individual graphemes in spelling to dictation (Rapp et al., 2002).

As explained earlier, the dual-route view of spelling to dictation holds that two routes are involved in parallel during the generation of word spellings. The nonlexical route activates individual graphemes by using high-probability phoneme-grapheme mappings, whereas the lexical route activates individual graphemes by retrieving whole-word lexical representations in the orthographic lexicon on the basis of the semantic specifications activated from the phonological lexeme level. The integration of the outputs generated by the two routes takes place at the level of individual graphemes. The lexical route is given a stronger weight over the nonlexical route in the generation of correct spellings due to the presence of feedback connections from the individual grapheme level to the lexeme level that serve to stabilize and amplify the lexical contribution. The individual graphemes of words with a dense neigh- borhood receive more activation than words with a sparse neighborhood, because the former receive activation from many more words than the latter. To provide an illustration of how the feedback mechanism works, take the word cat presented for spelling to dictation. After the activation and selection of its phonological lexeme, the orthographic lexeme is activated on the basis of semantic specifications. The latter, in turn, will partially activate the individual graphemes that constitute it $(c+a+t)$. The activated grapheme nodes $(c, a, t)$ then feed activation back to all the orthographic lexemes that contain those graphemes (e.g., "bat," "mat"). These orthographically related word forms will, in turn, send activation back down to the grapheme nodes, thereby increasing the activation of those shared grapheme nodes. Similarly, cyclic feedforward and feedback processing creates positive feedback loops between an orthographic lexical representation and its individual graphemes. Therefore, words with dense neighborhoods receive activation from many similar formal words via the shared individual graphemic units, unlike words with sparse neighborhoods, which receive activation from only a small number of similar, formal words.

It could be argued that the neighborhood effects reported here do not occur at the level of orthographic representations (between orthographic lexemes and individual graphemes), but at the level of phonological representations, since certain orthographic neighbors are also phonological neighbors. If this were true, however, our findings would tell us nothing about the interactivity issue in spelling to dictation. However, phonological neighborhood effects in auditory lexical decision and shadowing tasks have been found to be inhibitory rather than facilitatory (e.g., Luce \& Pisoni, 1998; Vitevitch, 2002; Vitevitch \& Luce, 1998, 1999, in English; Ziegler \& Muneaux, 2007, in French). Moreover, in the control shadowing task, the neighborhood effect was virtually nonexistent. Finally, a regression analysis was run on the spelling latencies with the number of phonographic neighbors, phonological neighbors, orthographic neighbors, and acoustic duration entered as factors. The number of orthographic neighbors $(\beta=$ $-.32, t=-2.54, p<.01)$ and acoustic duration $(\beta=$ $.42, t=3.40, p<.01)$ had a reliable influence on spelling latencies, whereas number of phonographic neighbors $(\beta=-.19, t=1.32, p>.05)$ and phonological neighbors $(\beta=-.24, t=1.68, p>.05)$ had no significant influence. We therefore feel confident that the neighborhood effects reported here originate at the level of individual graphemes and orthographic lexemes.

It could be argued that feedback from graphemes to orthographic lexemes is not necessary to account for the neighborhood facilitation effect found here if we consider a cognitive spelling architecture in which activation cascades throughout. Importantly, such an architecture is certainly not incompatible with the dual-route view of spelling, and could be easily accommodated within the Rapp et al. (2002) claim. If words that are phonological neighbors of the target (e.g., "hat" or "rat" for "cat") are activated in the phonological input lexicon, activation for those words is sent downstream in the processing system. Thus, the orthographic lexemes of rat and hat may have 
a high activation level, which in turn, cascades down to the grapheme level. However, since the number of phonological neighbors was controlled in our study, and the control shadowing task did not reveal a neighborhood effect, the facilitatory effect found on the oral spelling latencies is difficult to account for in terms of cascading activation from phonological neighbors; it could still be argued that the frequency of the orthographic neighbors is responsible for the spelling latency difference. Words from dense neighborhoods might cause more lexical activation in the lexical route than words from sparse neighborhoods because of their high-frequency neighbors, even if the number of phonological neighbors was controlled between the two types of items. However, a regression analysis performed on the latencies with the cumulative frequency of neighbors included as a factor (together with the number of phonographic neighbors, number of orthographic neighbors, acoustic duration) did not reveal a reliable influence of this factor $(\beta=-.05, t<1)$.

Finally, instead of appealing to the idea of a feedback mechanism to account for neighborhood effects in spelling, one may well ask whether these effects are the result of the operation of the nonlexical route. If words with many orthographic neighbors tend to have more frequent sublexical correspondences than do words with few neighbors, the phoneme-to-grapheme conversion procedure should operate more rapidly on the former than on the latter. It should be remembered that the consistency of the sublexical units of the words was controlled for; but what about the frequency of sublexical correspondences? A close examination of the frequency of the sublexical correspondence at the onset, vowel, coda, and rime levels revealed that the two sets of items were matched for the onset and coda frequency but differed reliably at the level of vowel and rime frequency. However, regression analyses performed on the spelling latencies did not reveal a reliable influence of either the vowel $(\beta=.19, t=1.2$, n.s. $)$ or the rime $(\beta=-.05, t<1)$ frequency.

To conclude, we have reported a neighborhood facilitatory effect on spelling-to-dictation speed that accords nicely with the hypothesis that the cognitive spelling system has an interactive architecture that incorporates grapheme-to-lexeme feedback.

\section{AUTHOR NOTE}

The authors thank two anonymous reviewers for helpful comments. Correspondence concerning this article should be addressed to P. Bonin, LAPSCO/CNRS UMR 6024, Université Blaise Pascal, 34 avenue Carnot, B.F. 185, 63037 Clermont-Ferrand cedex, France (e-mail: patrick .bonin@univ-bpclermont.fr).

\section{REFERENCES}

ANDREws, S. (1997). The effect of orthographic similarity on lexical retrieval: Resolving neighborhood conflicts. Psychonomic Bulletin \& Review, 4, 439-461.

Cohen, J. D., MacWhinney, B., Flatt, M., \& Provost, J. (1993).

PsyScope: A new graphic interactive environment for designing psy- chology experiments. Behavior Research Methods, Instruments, \& Computers, 25, 257-271.

Coltheart, M., Davelaar, E., Jonasson, J. T., \& Besner, D. (1977). Access to the internal lexicon. In S. Dornic (Ed.), Attention and performance VI (pp. 535-555). Hillsdale, NJ: Erlbaum.

Dufour, S., Peereman, R., Pallier, C., \& Radeau, M. (2002). VoCoLex: une base de données lexicales sur les similarités phonologiques entre les mots français [VoCoLex: A lexical database on phonological similarity between French words]. L'Année Psychologique, 102, 725-746.

KreINER, D. S. (1996). Effects of word familiarity and phoneme-tographeme polygraphy on oral spelling time and accuracy. Psychological Record, 46, 49-70.

Lété, B., Sprenger-Charolles, L., \& Colé, P. (2004). MANUleX: A grade-level lexical database from French elementary-school readers. Behavior Research Methods, Instruments, \& Computers, 36, 156-166.

Luce, P. A., \& Pisoni, D. B. (1998). Recognizing spoken words: The neighborhood activation model. Ear \& Hearing, 19, 1-36.

McCloskey, M., Macaruso, P., \& Rapp, B. (2006). Grapheme-tophoneme feedback in the spelling system: Evidence from dysgraphia. Cognitive Neuropsychology, 23, 278-307.

New, B., Pallier, C., Ferrand, L., \& Matos, R. (2001). Une base de données lexicales du français contemporain sur Internet: LEXIQUE. L'Année Psychologique, 101, 447-462.

Peereman, R., \& Content, A. (1997). Orthographic and phonological neighborhoods in naming: Not all neighbors are equally influential in orthographic space. Journal of Memory \& Language, 37, 382-410.

Peereman, R., \& Content, A. (1999). LeXOP: A lexical database providing orthography-phonology statistics for French monosyllabic words. Behavior Research Methods, Instruments, \& Computers, 31, 376-379.

RAPP, B. (2002). Uncovering the cognitive architecture of spelling. In A. Hillis (Ed.), Handbook on adult language disorders: Integrating cognitive neuropsychology, neurology, and rehabilitation (pp. 47-70). Philadelphia: Psychology Press.

Rapp, B., Epstein, C., \& Tainturier, M.-J. (2002). The integration of information across lexical and sublexical processes in spelling. Cognitive Neuropsychology, 19, 1-29.

TAINTURIER, M.-J., \& RAPP, B. (2001). The spelling process. In B. Rapp (Ed.), Handbook of cognitive neuropsychology: What deficits reveal about the human mind (pp. 263-289). Philadelphia: Psychology Press.

Vitevitch, M. S. (2002). Naturalistic and experimental analyses of word frequency and neighborhood density effects in slips of the ear. Language \& Speech, 45, 407-434.

Vitevitch, M. S., \& LuCE, P. A. (1998). When words compete: Levels of processing in spoken word perception. Psychological Science, $\mathbf{9}$, 325-329.

Vitevitch, M. S., \& Luce, P. A. (1999). Probabilistic phonotactics and neighborhood activation in spoken word recognition. Journal of Memory \& Language, 40, 374-408.

Vitevitch, M. S., \& Stamer, M. K. (2006). The curious case of competition in Spanish speech production. Language \& Cognitive Processes, 21, 760-770.

Yates, M. (2005). Phonological neighbors speed visual word processing: Evidence from multiple tasks. Journal of Experimental Psychology: Learning, Memory, \& Cognition, 31, 1387-1397.

Ziegler, J. C., \& Muneaux, M. (2007). Orthographic facilitation and phonological inhibition in spoken word recognition: A developmental study. Psychonomic Bulletin \& Review, 14, 75-80.

\section{NOTE}

1. When the data were analyzed by subtracting stimulus duration from each response time, the pattern of results was similar.

(Manuscript received June 4, 2008; revision accepted for publication October 9,2008 .) 